\title{
Decrease in sleep quality during COVID-19 outbreak
}

\author{
Adriano D. S. Targa ${ }^{1,2} \cdot$ Iván D. Benítez ${ }^{1,2} \cdot$ Anna Moncusí-Moix ${ }^{1,2} \cdot$ Maria Arguimbau $^{1,2} \cdot$ Jordi de Batlle ${ }^{1,2}$. \\ Mireia Dalmases ${ }^{1,2} \cdot$ Ferran Barbé $^{1,2,3}$
}

Received: 5 August 2020 / Revised: 14 September 2020 / Accepted: 22 September 2020 / Published online: 29 September 2020

(C) Springer Nature Switzerland AG 2020

\begin{abstract}
Purpose The COVID-19 outbreak witnessed in the first months of 2020 has led to unprecedented changes in society's lifestyles. In the current study, we aimed to investigate the effect of this unexpected context on sleep.

Methods During the COVID-19 outbreak, we performed an online survey with individuals formerly recruited for validation of the Spanish version of the sleep questionnaire Satisfaction, Alertness, Timing, Efficiency, and Duration (SATED). In the current survey, we asked the participants to complete the previously answered questionnaires including the Pittsburgh Sleep Quality Index (PSQI), a modified version of the Epworth Sleepiness Scale (ESS), and the SATED questionnaire. We also assessed the mood by the Profile of Mood States (POMS) questionnaire.

Results The 71 participants were mostly women $(75 \%)$ with a mean $( \pm \mathrm{SD})$ age of $40.7 \pm 11.9$ years. Comparing the previous PSQI score to that during the COVID-19 outbreak, we observed worsening sleep quality ( $5.45 \pm 3.14$ to $6.18 \pm 3.03$ points, $p=$ $0.035)$. In parallel, there was an increase in the negative mood $(p=0.002)$. Accordingly, the decrease in sleep quality was substantially correlated with negative mood $(p<0.001)$. There were no differences in the ESS or SATED.

Conclusions The COVID-19 outbreak-associated events correlate with decreased sleep quality in association with an increase in negative mood. Considering the importance of sleep for a healthy life, and in particular for immune function, efforts should be made to improve awareness on this matter and to offer psychological assistance to affected individuals.
\end{abstract}

Keywords COVID-19 $\cdot$ Restrictive measures $\cdot$ Sleep $\cdot$ Mood

\section{Abbreviations \\ BMI Body mass index \\ ESS Modified Epworth Sleepiness Scale \\ POMS Profile of Mood States}

Adriano D. S. Targa and Iván D. Benítez contributed equally to this work.

Adriano D. S. Targa and Iván D. Benítez are co-first authors.

Electronic supplementary material The online version of this article (https://doi.org/10.1007/s11325-020-02202-1) contains supplementary material, which is available to authorized users.

Ferran Barbé

febarbe.lleida.ics@gencat.cat

1 Translational Research in Respiratory Medicine, Hospital Universitari Arnau de Vilanova-Santa Maria, IRBLleida, Lleida, Spain

2 Centro de Investigación Biomédica en Red de Enfermedades Respiratorias (CIBERES), Madrid, Spain

3 Respiratory Department, Hospital Universitari Arnau de Vilanova, Rovira Roure, 80, 25198 Lleida, Spain
PSQI Pittsburgh Sleep Quality Index

SATED Satisfaction, Alertness, Timing, Efficiency, and Duration

\section{Introduction}

The COVID-19 outbreak witnessed in the first months of 2020 led to unprecedented changes in society's lifestyle. In a tentative to counteract the growing number of positive cases and avoid the health care system collapse, most of the world leaders determined the home confinement as the most effective measure to be followed. In Spain, restrictions on the movement of individuals started on March 14, exceeding 2 months up to this date (and likely to be extended). As a consequence, the population was compelled to adjust the personal and professional life to this condition, working from home, home teaching their children, and reducing social interaction.

Sleep is an important and highly susceptible behavior within this context. The flexibility in schedules due to social restrainment led to changes in the wake-up time and bedtime. 
Accordingly, recent studies reported that individuals are waking up and sleeping later during the confinement, possibly influenced by the increased use of digital media near bedtime [1]. Also, reduced exposure to the sunlight, limited activity during the day, and alterations in the food time may lead to dysregulation in the circadian rhythms and, in consequence, may affect sleep $[2,3]$. Furthermore, sleep quality is closely related to the mood, which was demonstrably altered during this time $[4,5]$. Huang and collaborators (2020) observed a high prevalence of generalized anxiety disorder which seemed to be associated with the time spent focusing on the COVID-19 ( $\geq 3 \mathrm{~h}$ per day) [6]. In addition, in a study performed during the initial stage of the COVID-19 outbreak in China, 54\% of participants rated the psychological impact as moderate or severe and about onethird reported moderate-to-severe anxiety [7]. Accordingly, the few studies evaluating sleep during this period also evaluated anxiety, depression, or stress. Xiao and collaborators (2020) reported an association between anxiety and poor sleep quality assessed by the Pittsburgh Sleep Quality Index (PSQI) questionnaire [4]. Similarly, poor quality sleep was demonstrably increased in those with depression, anxiety, and stress [1]. However, proper evaluation of the effect of the COVID-19 outbreak on sleep is hindered by the design of the available studies. Considering this, we conducted an online survey from April 28, 2020, to May 12, 2020, (during COVID-19 outbreak) with individuals that had previously answered the same survey (preCOVID-19 period). The survey included the PSQI, a modified version of the Epworth Sleepiness Scale (ESS), the Satisfaction Alertness Timing Efficiency Duration (SATED), and the Profile of Mood States (POMS) questionnaire.

\section{Methods}

\section{Study population}

Our population was composed by part of the individuals recruited as an independent sample aiming to validate the Spanish version of the SATED questionnaire [8]. The participants were older than 18 years of age and considered to be physically and mentally able to participate in the study. The original sample was stratified by sex, age, and educational and socioeconomic level to properly represent the general population (for a detailed description, see [8]). This study was approved by the Clinical Research Ethics Committee of the Arnau de Vilanova University Hospital in Lleida (CEIC1694), and conducted according to the principles outlined by the Declaration of Helsinki.

\section{Study design}

The population was first recruited in 2017 as an independent sample to validate the Spanish version of the SATED questionnaire [8] (Fig. 1). Clinical and sociodemographic data were collected, and the participants completed the PSQI, ESS, SATED, and POMS questionnaires. During the COVID-19 outbreak in Spain, the individuals were contacted by their electronic addresses and asked to complete the previously answered questionnaires. The survey was available for a limited time window (from April 28 to May 12, 2020) and we obtained the answer from 71 individuals. Clinical and sociodemographic data were collected again due to possible changes over the years.

\section{Clinical and sociodemographic variables}

The following variables were collected: age, sex, educational level, work schedule, physical activity, previous diseases, medication intake, alcohol consumption, smoking, and caffeine-based drinks ingestion. Body mass index (BMI) was calculated as body weight (in $\mathrm{kg}$ )/height (in $\mathrm{m}^{2}$ ).

\section{Pittsburgh Sleep Quality Index}

The sleep quality was assessed by the PSQI [9]. The questionnaire was composed of 19 questions representing one of the seven components of sleep quality: subjective sleep quality, sleep latency, sleep duration, sleep efficiency, sleep disturbance, sleep medication intake, and daytime dysfunction. Each component score was rated on a 3-point scale, leading to a sum of up to 21 points. A PSQI score $>5$ indicated a poor sleep quality whereas a PSQI score $\leq 5$ indicated a good sleep quality.

200 individuals were assessed for their eligibility in 2017 (validation of the Spanish version of the SATED questionnaire)

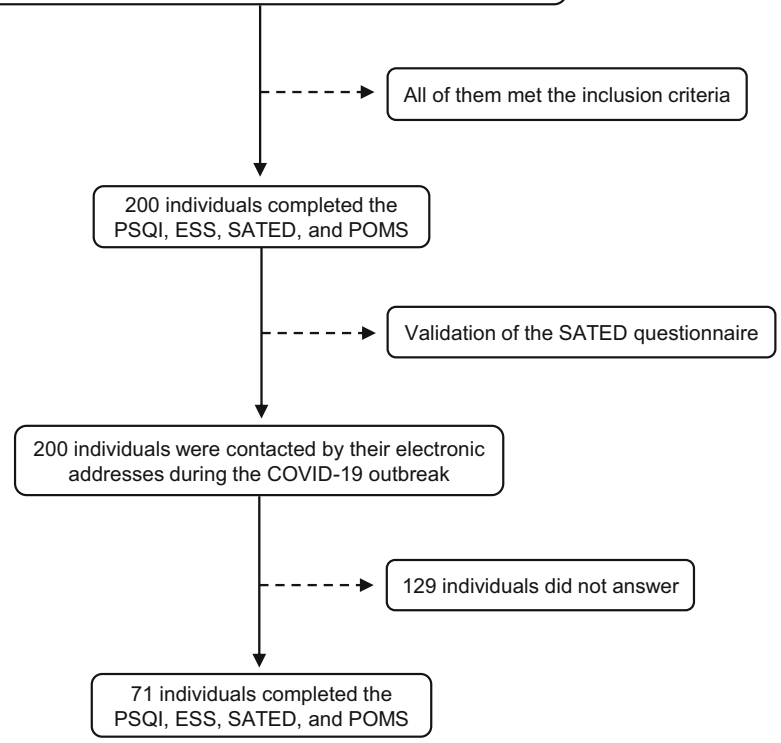

Fig. 1 Flow chart. ESS, Modified Epworth Sleepiness Scale; POMS, Profile of Mood States; PSQI, Pittsburgh Sleep Quality Index; SATED, Satisfaction Alertness Timing Efficiency Duration 


\section{Modified Epworth Sleepiness Scale}

The excessive daytime somnolence was assessed by the ESS [10]. The questionnaire is originally composed of 8 questions to assess the chance of falling asleep during different daily situations. Three questions that were considered inappropriate due to the restrictive measures were excluded. Each question was rated on a 3-point scale, in which 0 represented no chance of occurrence, and 3 indicated a high chance of occurrence. The overall score ranged from 0 to 15 points. Higher scores represented increased daytime somnolence.

\section{Satisfaction Alertness Timing Efficiency Duration}

Sleep health was further assessed by the SATED [8]. The questionnaire was composed of 5 questions representing one of the 5 following sleep-related dimensions: subjective satisfaction, alertness during waking hours, appropriate timing, efficiency, and duration. Each question was rated on a 2point scale, leading to a sum of up to 10 points. Higher scores indicated better sleep health.

\section{Profile of mood states}

The mood was assessed by the POMS [11]. The questionnaire was composed of 28 questions representing one of the 5 following dimensions: tension ( 5 questions), depression (6 questions), anger (7 questions), vigor (6 questions), and fatigue (4 questions). Each question was rated on a 5-point scale, with 0 representing "not at all" and 4 indicating "extremely." The score of each dimension was the sum of the given rates for each of the corresponding questions. The positive subscale corresponded to "vigor" and the negative subscale was the sum of tension, depression, anger, and fatigue. The total score was calculated by subtracting the positive subscale from the total of the negative subscale $(+100$, to avoid negative values). Thus, higher scores indicated a negative mood.

\section{Statistical analysis}

The means (standard deviation, SD) were estimated for quantitative variables and the absolute and relative frequencies were used for qualitative variables. We compared the questionnaires' outcomes between both periods (pre- and during COVID-19 outbreak) using $t$ test or Wilcoxon rank sum test for paired samples. Furthermore, the relationship between POMS and PSQI scores during the COVID-19 outbreak was assessed through Spearman's rank correlation coefficient. Finally, the differences in the PSQI components according to the working condition were assessed by linear models. All statistical analyses and data processing procedures were performed using R software, version 3.5.2 (Vienna, Austria).

\section{Results}

\section{Participants' characteristics}

The 71 participants were mostly women $(75 \%)$ with a mean $( \pm$ SD) age of $40.7 \pm 11.9$ years old and a mean $( \pm$ SD) BMI of $23.0 \pm 3.7 \mathrm{~kg} \mathrm{~m}^{-2}$ (Table 1). Only $1(1 \%)$ individual had been diagnosed with COVID-19 by the time of the survey and no positive case at the same home was reported. The working condition was altered in most of the cases, with $32(45 \%)$ individuals working from home and $12(17 \%)$ unemployed due to the lockdown whilst $16(23 \%)$ remained working at the workplace and $10(14 \%)$ were already unemployed before the COVID-19 outbreak.

\section{COVID-19 outbreak, sleep, and mood}

According to the PSQI, there was a decrease in sleep quality during this period, demonstrated by a mean $( \pm \mathrm{SD})$ change of $0.73 \pm 3.01(p=0.035)$ between the baseline evaluation and that during the COVID-19 outbreak (Table 2). A similar outcome was observed in relation to sleep latency, with a mean ( \pm SD) change of $0.27 \pm 0.96(p=0.028)$ between the two timepoints. Differently, we did not observe changes related to the excessive daytime somnolence and other sleep-related aspects

Table 1 Characteristics of the participants

Global

$n=71$

$n(\%)$, mean $(\mathrm{SD})$

Sociodemographic data

$\begin{array}{ll}\text { Sex, woman } & 53(75 \%) \\ \text { Age, years } & 40.7(11.9) \\ \text { BMI, kg m }{ }^{-2} & 23.0(3.7) \\ \text { Education, years } & 9.0(2.2) \\ \text { Health condition } & \\ \text { Rhinitis } & 5(7 \%) \\ \text { Hypo-thyroidism } & 4(6 \%) \\ \text { Hypertension } & 3(4 \%) \\ \text { COVID-19 } & \\ \text { Diagnosis } & 1(1 \%) \\ \text { Hospital admission } & 0(0 \%) \\ \text { Household cases } & 0(0 \%) \\ \text { Working condition } & \\ \text { Workplace } & 16(23 \%) \\ \text { Home working } & 32(45 \%) \\ \text { Unemployed due to lockdown } & 12(17 \%) \\ \text { Previously unemployed } & 10(14 \%)\end{array}$

$B M I$, body mass index; $n$, number; $S D$, standard deviation 
Table 2 COVID-19 outbreak and sleep

\begin{tabular}{lllrl}
\hline & $\begin{array}{l}\text { Pre-COVID-19 } \\
n=71 \\
\text { Mean (SD) }\end{array}$ & $\begin{array}{l}\text { COVID-19 } \\
n=71 \\
\text { Mean (SD) }\end{array}$ & $\begin{array}{l}\text { Change } \\
\text { Mean (SD) }\end{array}$ & $p$ value \\
\hline PSQI & $5.45(3.14)$ & $6.18(3.03)$ & $0.73(3.01)$ & 0.035 \\
Subjective sleep quality & $1.13(0.67)$ & $1.20(0.67)$ & $0.07(0.78)$ & 0.442 \\
Sleep latency & $1.07(0.90)$ & $1.34(0.92)$ & $0.27(0.96)$ & 0.028 \\
Sleep duration & $0.83(0.61)$ & $0.72(0.66)$ & $-0.11(0.75)$ & 0.207 \\
Sleep efficiency & $0.34(0.77)$ & $0.55(0.82)$ & $0.21(0.91)$ & 0.090 \\
Sleep disturbance & $1.21(0.56)$ & $1.20(0.43)$ & $-0.01(0.62)$ & 0.860 \\
Sleep medication intake & $0.24(0.73)$ & $0.35(0.83)$ & $0.11(0.77)$ & 0.283 \\
Daytime dysfunction & $0.63(0.68)$ & $0.83(0.79)$ & $0.2(0.84)$ & 0.057 \\
ESS & $6.23(2.64)$ & $5.75(2.99)$ & $-0.48(2.33)$ & 0.127 \\
SATED & $7.25(1.95)$ & $7.70(1.99)$ & $0.45(2.44)$ & 0.110 \\
\hline
\end{tabular}

ESS, Modified Epworth Sleepiness Scale; PSQI, Pittsburgh Sleep Quality Index; SATED, Satisfaction Alertness Timing Efficiency Duration; $S D$, standard deviation as indicated by the ESS $(p=0.127)$ and $\operatorname{SATED}(p=0.110)$, respectively.

The negative mood was significantly increased during the COVID-19 outbreak as demonstrated by a mean ( \pm SD) change of $6.27 \pm 14.92(p=0.002)$ between the two timepoints in the POMS total score (Table 3). Accordingly, there was an increase in the negative subscale with a mean $( \pm$ SD) change of $5.63 \pm 13.88(p=0.001)$ as a result of increases in tension [mean $( \pm \mathrm{SD})$ change: $1.9 \pm 4.38 ; p=0.001]$, depression [mean $( \pm \mathrm{SD})$ change: $1.17 \pm 4.15 ; p=0.017$ ], and anger [mean $( \pm \mathrm{SD})$ change: $2.07 \pm 5.88 ; p=0.002$ ]

\section{Correlations between sleep quality and mood}

To evaluate whether the observed decrease in sleep quality was associated with the increase in the negative mood, we investigated the correlations between PSQI and POMS questionnaires (Table 4). There was substantial correlation among POMS total score and different PSQI items such as PSQI total score (corr $=0.55, p<0.001$ ), subjective sleep quality (corr $=$ $0.49, p<0.001$ ), sleep latency (corr $=0.31, p=0.008$ ), sleep duration (corr $=0.33, p=0.005$ ), sleep medication intake (corr $=0.24, p=0.040$ ), and daytime dysfunction (corr $=0.49, p<$ 0.001). Similarly, distinct correlations were observed among PSQI items and the specific dimensions of POMS.

\section{Discussion}

In the current study, we investigated the influence of the COVID-19 outbreak-associated events on sleep. According to the PSQI, there was a decrease in sleep quality during this period, possibly associated with an increase in sleep latency. In parallel, the mood state evaluation indicated an increase in the score of dimensions representing a negative mood such as tension, depression, and anger. Accordingly, there was substantial correlation between sleep quality and mood states. On the other hand, we did not observe any differences in relation
Table 3 COVID-19 outbreak and $\operatorname{mood}$

\begin{tabular}{lllll}
\hline & $\begin{array}{l}\text { Pre-COVID-19 } \\
n=71 \\
\text { Mean (SD) }\end{array}$ & $\begin{array}{l}\text { COVID-19 } \\
n=71 \\
\text { Mean (SD) }\end{array}$ & $\begin{array}{l}\text { Change } \\
\text { Mean (SD) }\end{array}$ & $p$ value \\
\hline POMS & $107(17.5)$ & $113(17.3)$ & $6.27(14.92)$ & 0.002 \\
Tension & $5.46(4.35)$ & $7.37(4.16)$ & $1.9(4.38)$ & 0.001 \\
Depression & $3.79(4.52)$ & $4.96(3.99)$ & $1.17(4.15)$ & 0.017 \\
Anger & $5.14(6.39)$ & $7.21(5.87)$ & $2.07(5.88)$ & 0.002 \\
Vigor & $12.5(5.25)$ & $11.8(5.31)$ & $-0.63(5.65)$ & 0.388 \\
Fatigue & $4.76(3.61)$ & $5.25(3.92)$ & $0.49(3.39)$ & 0.110 \\
Positive subscale & $12.5(5.25)$ & $11.8(5.31)$ & $-0.63(5.65)$ & 0.388 \\
Negative subscale & $19.2(16.3)$ & $24.8(15.2)$ & $5.63(13.88)$ & 0.001 \\
\hline
\end{tabular}

POMS, Profile of Mood States; $S D$, standard deviation 
Table 4 Correlations between sleep quality and mood

POMS

\begin{tabular}{|c|c|c|c|c|c|c|c|c|}
\hline & Tension & Depression & Anger & Vigor & Fatigue & Positive & Negative & Total Score \\
\hline \multicolumn{9}{|l|}{ PSQI } \\
\hline Total score & $0.44 * * *$ & $0.38 * *$ & $0.31 * *$ & $-0.39 * * *$ & $0.46^{* * *}$ & $-0.39 * * *$ & $0.47 * * *$ & $0.55^{* * *}$ \\
\hline Subjective sleep quality & $0.41 * * *$ & $0.31 * *$ & $0.34 * *$ & $-0.31 * *$ & $0.42 * * *$ & $-0.31 * *$ & $0.44 * * *$ & $0.49 * * *$ \\
\hline Sleep latency & 0.21 & 0.12 & 0.18 & $-0.38 * *$ & 0.20 & $-0.38 * *$ & 0.21 & $0.31 * *$ \\
\hline Sleep duration & $0.29 *$ & $0.33 * *$ & 0.17 & -0.09 & $0.25 *$ & -0.09 & $0.30 *$ & $0.33 * *$ \\
\hline Sleep efficiency & 0.16 & 0.14 & 0.02 & -0.10 & 0.18 & -0.10 & 0.17 & 0.17 \\
\hline Sleep disturbance & $0.26^{*}$ & 0.15 & -0.01 & -0.05 & 0.23 & -0.05 & 0.18 & 0.17 \\
\hline Sleep medication intake & 0.21 & $0.29 *$ & 0.15 & -0.09 & 0.13 & -0.09 & $0.26^{*}$ & $0.24 *$ \\
\hline Daytime dysfunction & $0.27 *$ & $0.34 * *$ & $0.30 * *$ & $-0.47 * * *$ & $0.48^{* * *}$ & $-0.47 * * *$ & $0.39 * * *$ & $0.49 * * *$ \\
\hline
\end{tabular}

${ }^{*} p<0.05, * * p<0.01, * * * p<0.001 . P S Q I$, Pittsburgh Sleep Quality Index

to the daytime somnolence as indicated by the ESS nor on the sleep dimensions assessed by the SATED questionnaire.

The findings herein presented seem to confirm the available data in the literature. Cellini and collaborators (2020) evaluated 1310 individuals living in the Italian territory and reported a decrease in sleep quality, which was stronger in individuals with higher symptoms of depression, anxiety, and stress [1]. Similarly, Xiao and collaborators (2020) demonstrated that the decrease in sleep quality in individuals confined at home for 14 days in central China was associated with an increase in anxiety and stress [4]. Although those outcomes are headed towards the same direction to the ones observed in this study, proper conclusions were hindered by limitations related to these studies' designs. Here, we compared an evaluation before the COVID-19 outbreak to that during this period, which confirmed a deleterious effect of this context on sleep quality. Accordingly, there was a substantial correlation between sleep-related parameters and mood. The most affected parameter in the PSQI was the sleep latency, which presented a negative correlation with the positive subscale of POMS, i.e., the lack of positive mood appeared to be associated with increased sleep latency. In fact, the PSQI total score presented a positive correlation with all the parameters of POMS that composed the negative subscale, such as tension, depression, anger, and fatigue.

Other factors may have accounted for the observed decrease in sleep quality in addition to the negative mood. Changes in social- and work-related schedules lead to alterations in sleep. We did not observe an influence of the working condition on sleep quality (see Table S1 in the supplemental material); however, recent studies reported that people are waking up and sleeping later during this period [1]. Although this may be due to late chronotype, such situation could be related to the reported increased use of digital media near bedtime associated with the COVID-19 outbreak [1, 12]. In fact, the changes in sleep latency here presented may be a consequence of excessive screen time at night. Furthermore, the imposed restrictions lead to a decrease in sunlight exposure and physical activity, which are important factors for circadian rhythm maintenance [3, 13, 14]. Accordingly, these behaviors altogether lead to a disruption of circadian rhythms, further aggravating sleep behavior, and mood $[15,16]$. Also, such disturbance may affect the immune response [17], body temperature [18], blood pressure [19], metabolism, and energy homeostasis [20].

It is important to address that although the original sample was stratified by sex, age, and educational and socioeconomic level to properly represent the general population, our sample was reduced to the individuals who answered the questionnaires during the COVID-19 outbreak. Accordingly, our population was mostly composed of women with a mean age of approximately 40 years. Studies showed that women are more susceptible to worry and to increased psychological burden $[21,22]$. In addition, schedules, screen time at night, and the perception of the context may be distinct across the different ages. Thus, the results herein presented should be taken with caution, especially when making generalizations to a broader population. Besides this, this study has some limitations. Considering the influence of age on sleep quality, the 3 years of difference between baseline and follow-up evaluations may have affected the sleep quality, in addition to the context of the COVID-19 outbreak. Also, although we have considered alterations in health conditions, changes in medication, differences in food and drink habits during this period, other modifications in lifestyle may have contributed to the observed outcomes. Our study has some strengths as well. Sleep was evaluated by different validated questionnaires. In addition, we investigated the influence of the COVID-19 outbreakassociated events on sleep with a baseline assessment, when the individuals were not under this context.

In summary, we observed a decrease in sleep quality during the COVID-19 outbreak according to the PSQI. In parallel, 
the mood state evaluation indicated an increase in the scores of dimensions representing a negative mood such as tension, depression, and anger. Accordingly, the decrease in sleep quality was substantially correlated with negative mood. Considering the importance of sleep for a healthy life, and in particular for the immune function, efforts should be made to improve awareness on this matter and to psychologically assist the individuals during this period. These findings unravel a possible facet associated with the restrictive measures, which should be considered in case of future situations.

Acknowledgments We would like to express our gratitude to all the individuals who answered the questionnaires.

Authors' contributions Adriano Targa: conceptualization, methodology, writing - original draft/review and editing. Iván D. Benítez: conceptualization, methodology, data curation, formal analysis, writing - review and editing. Anna Moncusí-Moix: conceptualization, methodology, data curation, writing - review and editing. Maria Arguimbau: methodology, writing - review and editing. Jordi de Batlle: writing - review and editing. Mireia Dalmases: conceptualization, writing - review and editing. Ferran Barbé: conceptualization, writing - review and editing.

Data availability All data support our published claims and comply with field standards.

\section{Compliance with ethical standards}

Conflict of interest The authors declare that they have no conflict of interest.

Ethics approval This study was performed in line with the principles of the Declaration of Helsinki. Approval was granted by the Clinical Research Ethics Committee of the Arnau de Vilanova University Hospital in Lleida (CEIC-1694).

Consent to participate The individuals signed an informed consent form to participate.

Consent to publish The individuals signed an informed consent form to publish.

Code availability Not applicable.

\section{References}

1. Cellini N, Canale N, Mioni G, Costa S (2020) Changes in sleep pattern, sense of time and digital media use during COVID-19 lockdown in Italy. J Sleep Res 29. https://doi.org/10.1111/jsr.13074

2. da Silva FR, Guerreiro R d C, Andrade H d A et al (2020) Does the compromised sleep and circadian disruption of night and shiftworkers make them highly vulnerable to 2019 coronavirus disease (COVID-19)? Chronobiol Int 00:1-11. https://doi.org/10. 1080/07420528.2020.1756841

3. Van De Langenberg D, Vlaanderen JJ, Dollé MET et al (2019) Diet, physical activity, and daylight exposure patterns in night- shift workers and day workers. Ann Work Expo Health 63:9-21. https://doi.org/10.1093/annweh/wxy097

4. Xiao H, Zhang Y, Kong D et al (2020) Social capital and sleep quality in individuals who self-isolated for 14 days during the coronavirus disease 2019 (COVID-19) outbreak in January 2020 in China. Med Sci Monit 26:1-8. https://doi.org/10.12659/MSM. 923921

5. Liu N, Zhang F, Wei C, Jia Y, Shang Z, Sun L, Wu L, Sun Z, Zhou Y, Wang Y, Liu W (2020) Prevalence and predictors of PTSS during COVID-19 outbreak in China hardest-hit areas: gender differences matter. Psychiatry Res 287:112921. https://doi.org/10. 1016/j.psychres.2020.112921

6. Huang Y, Zhao N (2020) Generalized anxiety disorder, depressive symptoms and sleep quality during COVID-19 outbreak in China: a web-based cross-sectional survey. Psychiatry Res 288:112954. https://doi.org/10.1016/j.psychres.2020.112954

7. Wang C, Pan R, Wan X, Tan Y, Xu L, Ho CS, Ho RC (2020) Immediate psychological responses and associated factors during the initial stage of the 2019 coronavirus disease (COVID-19) epidemic among the general population in China. Int J Environ Res Public Health 17. https://doi.org/10.3390/ijerph17051729

8. Benítez I, Roure N, Pinilla L, Sapiña-Beltran E, Buysse DJ, Barbé F, de Batlle J (2020) Validation of the satisfaction, alertness, timing, efficiency and Duration (SATED) questionnaire for sleep health measurement. Ann Am Thorac Soc 17:338-343. https://doi.org/ 10.1513/AnnalsATS.201908-628OC

9. Buysse DJ, Reynolds CF, Monk TH et al (1989) The Pittsburgh sleep quality index: a new instrument for psychiatric practice and research. Psychiatry Res 28:193-213. https://doi.org/10.1016/ 0165-1781(89)90047-4

10. Johns MW (1992) Reliability and factor analysis of the Epworth Sleepiness Scale. Sleep. 15:376-381. https://doi.org/10.1093/sleep/ 15.4.376

11. Andrade E, Arce C, De Francisco C et al (2013) Abbreviated version in spanish of the poms questionnaire for adult athletes and general population. Rev Psicol del Deport 22(1):95-102.

12. Altena E, Baglioni C, Espie CA, Ellis J, Gavriloff D, Holzinger B, Schlarb A, Frase L, Jernelöv S, Riemann D (2020) Dealing with sleep problems during home confinement due to the COVID-19 outbreak: practical recommendations from a task force of the European CBT-I Academy. J Sleep Res 29:1-7. https://doi.org/ $10.1111 /$ jsr. 13052

13. Legates TA, Fernandez DC, Hattar S (2014) Light as a central modulator of circadian rhythms, sleep and affect. Nat Rev Neurosci 15:443-454

14. van Maanen A, Meijer AM, van der Heijden KB, Oort FJ (2016) The effects of light therapy on sleep problems: a systematic review and meta-analysis. Sleep Med Rev 29:52-62.

15. Dijk DJ, Czeisler CA (1995) Contribution of the circadian pacemaker and the sleep homeostat to sleep propensity, sleep structure, electroencephalographic slow waves, and sleep spindle activity in humans. J Neurosci 15:3526-3538. https://doi.org/10.1523/ jneurosci.15-05-03526.1995

16. Mcclung CA (2011) Circadian rhythms and mood regulation: insights from pre-clinical models. Eur Neuropsychopharmacol 21: S683-S693

17. Scheiermann C, Kunisaki Y, Frenette PS (2013) Circadian control of the immune system. Nat Rev Immunol 13:190-198

18. Refinetti $\mathrm{R}$ (2010) The circadian rhythm of body temperature. Front Biosci 15:564. https://doi.org/10.2741/3634

19. Parati G, Faini A, Valentini M (2006) Blood pressure variability: its measurement and significance in hypertension. Curr Hypertens Rep 8:199-204 
20. Panda S (2016) Circadian physiology of metabolism. Science 354: 1008-1015. https://doi.org/10.1126/science.aah4967

21. Barber SJ, Kim H (2020) COVID-19 worries and behavior changes in older and younger men and women. J Gerontol Ser B Psychol Sci Soc Sci. https://doi.org/10.1093/geronb/gbaa068

22. Lai J, Ma S, Wang Y, Cai Z, Hu J, Wei N, Wu J, du H, Chen T, Li R, Tan H, Kang L, Yao L, Huang M, Wang H, Wang G, Liu Z, Hu
S (2020) Factors associated with mental health outcomes among health care workers exposed to coronavirus disease 2019. JAMA Netw Open 3:e203976. https://doi.org/10.1001/jamanetworkopen. 2020.3976

Publisher's note Springer Nature remains neutral with regard to jurisdictional claims in published maps and institutional affiliations. 